Zagazig J. Agric. Res., Vol. 43 No. (3) 2016

http:/www.journals.zu.edu.eg/journalDisplay.aspx?Journalld=1\&queryType=Master

\title{
INFLUENCE OF PLANTING DENSITY AND NITROGEN FERTILIZER LEVELS ON FRESH FORAGE YIELD AND QUALITY OF SOME FORAGE SORGHUM GENOTYPES
}

\author{
Ahamed, M. Ibrahim ${ }^{1^{*}}$, E.M. Zeidan ${ }^{2}$, H.G.M. Gweifel ${ }^{2}$, I.M. Abd El-Hameed ${ }^{2}$ and \\ Sanaa A. Mahfouz ${ }^{1}$
}

1. Regional Cent. Food and Feed, ARC. Giza, Egypt

2. Agron. Dept., Fac. Agric., Zagazig Univ., Egypt

\begin{abstract}
Two field experiments were conducted during two successive summer growing seasons 2013 and 2014 in an administration field at Meet Gaber village, Belbais District, Sharkia Governorate, Egypt. The experiment aimed to study the effect of four planting densityies (D1:70000 plants/fad., D2: 105000 plants/fad., D3:140000 plants/fad.; D4: 210000 plants/fad.) and three nitrogen fertilizer levels (N1: $15 \mathrm{~kg} \mathrm{~N} /$ fad./cut; $\mathrm{N} 2: 30 \mathrm{~kg} \mathrm{~N} /$ fad./ cut; N3: $45 \mathrm{~kg} \mathrm{~N} /$ fad./cut) on fresh weight /plant, fresh forage yield/fad., as well as crude protein and fiber contents in leaves and stems at $1^{\text {st }}$ and $2^{\text {nd }}$ cut of three forage sorghum genotypes (G1: Sudan grass (Sorghum sudanense (Piper) Stapf, cv. Giza 2),G2: sweet sorghum (S. bicolor L. Moench, cv. Giza 1), and G3: sorghum (S. bicolor L. Moench) x Sudan grass (S. sudanense (Piper) Stapf, cv. Surdan) as an interspecies hybrid. The obtained results could be summarized as follows: Sudan grass was superior in fresh weight /plant, fresh forage yield /fad., where sweet sorghum was superior in crude protein and fiber contents. Using planting density 70000 plants/fad., had a significant increase in fresh weight /plant, crude protein and fiber content in leaves and stems. Fresh forage yield/fad., was significantly increased due to increasing planting density up to 140000 or 210000 plants/fad., at $1^{\text {st }}$ and $2^{\text {nd }}$ cut in both seasons and their combined analysis. The increase of $\mathrm{N}$ level up to $45 \mathrm{~kg} \mathrm{~N} /$ fad., caused a significant increase in the aforementioned characters, except crude fiber content which significantly decreased at $1^{\text {st }}$ and $2^{\text {nd }}$ cut in both seasons and their combined analysis.
\end{abstract}

Key words: Forage sorghum genotypes, planting density, nitrogen, sudan grass, sweet sorghum, crude protein, crude fiber.

\section{INTRODUCTION}

In Egypt, animal production is suffering scarcity because of the competition between the production of human food and animal feed. The insufficiency in forage production is more svere in summer season. Thus, increasing the forage production in the summer period is one of major target of the government to overcome the livestock production problem in order to face human needs.

Concerning the effect of forage sorghum genotypes, Almodares et al. (2009) stated that

\footnotetext{
* Corresponding author. Tel.: +201223639906

E-mail address: cropest@yahoo.com
}

fiber content of sweet sorghum was higher than corn. Sweet sorghum bagasse had lower crude protein and higher crude fiber than both corn and sweet sorghum. Ayub et al. (2010) showed that variety F-9603 of sorghum, significantly produced higher crude protein percentage $(7.62 \%)$ and lowest crude fiber percentage (28.37\%). Bozorgvar et al. (2013) showed that the sugar graze hybrid of sorghum produced the highest forage and dry matter yield. Mahmood et al. (2013) reported that sorghum cultivar 'Goliath' (Sorghum bicolor $\times$ S. bicolor hybrid) had a higher biomass yield than Bovital $(S$. 
bicolor $\times S$. sudanense hybrid) while, cultivar Bovital had a greater protein content than 'Goliath'. Soleymani and Shahrajabian (2013) pointed out that sweet sorghum cultivar Keller produced the maximum stalk, leaf and total dry matter than cultivar Rio.

Regarding plant density, El-Naggar (1983) found that increasing seeding rate decreased the percentage of protein in leaves, stalks and whole plant. Abd-Alla (1994) showed that increasing planting distance to $20 \mathrm{~cm}$ between hills decreased crude protein content in the plant and its parts. Yousef (2002) showed that fresh and dry weight/plant increased consistently and significantly as planting densities increased. Turgut et al. (2005) indicated that yield of sweet sorghum (Sorghum bicolor L. Moench) decreased with increasing intra-row spacing. Five- or $10 \mathrm{~cm}$ intra-row spacings gave more than 80 tonnes/ha., forage yield. Soleymani and Shahrajabian (2013) reported that the maximum stalk, leaf and total dry matter of sweet sorghum were achieved under planting density of 600000 plants/ha., Mahmood et al. (2013) showed that Planting density had no clear influence on most of the quality parameters of sorghum.

Abd-Alla (1994) and Bahrani and Deghani (2004) showed that increasing $\mathrm{N}$ levels $(0,40$, 60 and $80 \mathrm{~kg} \mathrm{~N} /$ fad.) increased percentage and production of crude protein and crude fiber. Almodares et al. (2009) evaluated the effects of four nitrogen treatments $(50,100,150$ and 200 $\mathrm{kg}$ urea/ha.) on crude protein and crude fiber contents of three fodders (corn, sweet sorghum and sweet sorghum bagasse), they showed that treatment of $200 \mathrm{~kg}$ urea/ha., had the highest protein content $(8 \%)$ and the lowest fiber content (31.90\%). Afzal et al. (2012) stated that addition of $\mathrm{N}$ up to $57.5 \mathrm{~kg} \mathrm{~N} / \mathrm{ha}$., enhanced significantly fresh and dry weights/plant, total green forage yield and total dry matter yield and crude protein content of sorghum. Abou-Amer and Kewan (2014) revealed that increasing nitrogen fertilizer levels up to $120 \mathrm{~kg} \mathrm{~N} /$ fad., had a significant increase in fodder yield (13.14 $\mathrm{t} /$ fad.), dry matter yield ( $2.12 \mathrm{t} /$ fad.) and crude protein $(11.45 \%)$, while crude fiber was the highest (37.08\%) as average of three cuts at 100 $\mathrm{kg} \mathrm{N}$ /fad., of sorghum (Sorghum bicolor L.). Hussein and Sabbour (2014) reported that increasing nitrogen fertilization up to 60 or 80 $\mathrm{kg} \mathrm{N} /$ fad., caused pronounced increases in fresh matter yield and dry matter yield of the $1^{\text {st }}$ and $2^{\text {nd }}$ cuts. Elshafey (2015) indicated that fresh and dry forage yields and crude protein content of sudan grass were significantly increased with increasing nitrogen level from 50 to 75 and 100 $\mathrm{kg} \mathrm{N} / \mathrm{fad}$., and significantly decreased due to increasing nitrogen rate from 100 to $125 \mathrm{~kg}$ $\mathrm{N} /$ fad., over both seasons. Therefore, the experiment aimed to study the effect of planting density and nitrogen fertilizer levels on fresh weight / plant, fresh forage yield /fad., as well as crude protein and fiber contents in leaves and stems at $1^{\text {st }}$ and $2^{\text {nd }}$ cut of three forage sorghum genotypes.

\section{MATERIALS AND METHODS}

Two field experiments were conducted during two successive summer growing seasons 2013 and 2014 in an administration field at Meet Gaber village, Belbais District, Sharkia Governorate, Egypt. This investigation was carried out to study the effect of four planting densities which were D1:70000 plants/fad., using planting distance of $20 \mathrm{~cm}$ on one side of the ridge and two plants/hill.; D2: 105000 plants/fad., using planting distance of $20 \mathrm{~cm}$ on one side of the ridge with three plants/hill.; D3:140000 plants/fad., using planting distance of $10 \mathrm{~cm}$ on one side of the ridge with two plants / hill and D4: 210000 plants / fad., using planting distance of $10 \mathrm{~cm}$ on one side of the ridge with three plants / hill. Also, to study the effect of three nitrogen fertilizer levels (N1: 15 $\mathrm{kg} \mathrm{N} /$ fad./cut; N2:30 kg N/fad./cut and N3: 45 $\mathrm{kg} \mathrm{N} /$ fad./cut) on fresh weight $\mathrm{g} /$ plant, fresh forage yield ton/fad., crude protein and fiber contents in leaves and stems at $1^{\text {st }}$ and $2^{\text {nd }}$ cuts of three forage sorghum genotypes (G1: Sudan grass (S. sudanense (Piper) Stapf, cv. Giza 2), G2: sweet sorghum (S. bicolor L.) Moench, cv. Giza 1), and G3: sorghum (S. bicolor L.) Moench) x Sudan grass (S. sudanense (Piper) Stapf, cv. Surdan) as an interspecies hybrid. The experiments were laid out in a split-split plot design in three replicates, where forage cultivars were assigned to the main plots and the planting densities occupied the first order sub plots. However, nitrogen fertilizer levels were allocated to the second order sub plots. The plot area was $10.5 \mathrm{~m}^{2}(2.5 \mathrm{~m}$ in length and $4.2 \mathrm{~m}$ in width) i.e., 7 ridges each of $60 \mathrm{~cm}$ width. 
Seeds (6 grains/hill) were sown on May $12^{\text {th }}$ for both summer successive seasons (2013 and 2014) with skillful workers. To obtain the four planting densities as mentioned above the thinning was made after three weeks from sowing to leave two or three plants/hill. The preceding crop was wheat (Triticum aestivum L.) and soil texture was clay in both seasons. Basal dose of $31 \mathrm{~kg} \mathrm{P}_{2} \mathrm{O}_{5} / \mathrm{fad}$., in form of calcium super phosphate was added at seedbed preparation, while potassium in the form of potassium sulphate $\left(48 \% \mathrm{~K}_{2} \mathrm{O}\right)$ was added in two equal doses, at seedbed preparation and two weeks after planting, whereas $\mathrm{N}$ fertilizer was added in the form of ammonium nitrate $33.5 \%$ $\mathrm{N})$ before the first irrigation and after the first and second cut. Weeds were controlled by hoewing. Other cultural practices were done as recommended in farmer fields.

\section{Recorded Data}

\section{Fresh farage weight/plant and fresh forage yield /fad.}

Fresh forage weight/plant and yield/fad., were recorded at cutting time (57 days after sowing for the first cut and 35 days later for the second cut). An area of $4.5 \mathrm{~m}^{2}$ (the inner three ridges of $2.5 \mathrm{~m}$ length $\times 60 \mathrm{~cm}$ width) was cut where, plots were hand clipped to a height of about $10 \mathrm{~cm}$ and weighted in $\mathrm{kg} / \mathrm{plot}$ to determine fresh forage yield, then converted to estimate fresh forage yield in ton/fad. Immediately after harvest, the whole plant dry matter and moisture content of all samples were determined by keeping $500 \mathrm{~g}$ of each sample in a laboratory drying oven set at a constant temperature of $105^{\circ} \mathrm{C}$ for 48 hours.

\section{Forage quality}

Crude protein content $(\mathrm{CP} \%)$ in leaves and stems was estimated with modified kjldahal method and multiplying the nitrogen content by factor of 5.75 to obtain the protein content (AOAC, 1990). Crude fiber content (CF\%) in leaves and stems was determined according to AOAC (1990).

\section{Statistical Analysis}

Statistical analysis of each experiment was performed as the methods outlined by Steel et al. (1997). Significance of differences between the various means of different characters under study were compared with the help of Duncan's multiple range test (1955). The combined analysis of variance was also computed for all traits recorded after establishing by Bartlett's homogeneity test, where the error variance of the individual season was homogeneous. In the interaction Tables, capital and small letters were used for comparison among row and column means, respectively.

\section{RESULTS AND DISCUSSION}

\section{Fresh Forage Weight / Plant and Forage Yield/fad.}

Tables 1 and 2 as well as Figs. 1, 2 and 3 show fresh weight / plant and fresh forage yield/fad., as affected by three forage sorghum genotypes, planting density and $\mathrm{N}$ fertilizer levels and their interactions at $1^{\text {st }}$ and $2^{\text {nd }}$ cut in both seasons and their combined analysis.

\section{Forage sorghum genotypes effect}

Results presented in Tables 1 and 2 indicate that the three forage sorghum genotypes varied significantly. These differences play a major role in determining not only a plant's ability to survive in the ecosystem, but also its potential productivity. Sudan grass the superem genotype in aforementioned traits, interspecies hybrid sorghum ranked the second and followed by sweet sorghum at $1^{\text {st }}$ cut in the $1^{\text {st }}$ and $2^{\text {nd }}$ seasons as well as in the combined analysis, while at $2^{\text {nd }}$ cut in the $1^{\text {st }}$ and $2^{\text {nd }}$ seasons as well as in the combined data, sudan grass was superior in aforementioned traits, sweet sorghum ranked the second and followed by interspecies hybrid sorghum. Higher fresh weight /plant and fresh forage yield/fad., of sudan grass were recorded by Almodares et al. (2006, 2009), Ayub et al. (2010), Afzal et al. (2012), Mahmood et al. (2013) and Soleymani and Shahrajabian (2013).

\section{Planting density effect}

It is evident from Tables 1 and 2 that the lowest Planting density (70000 plant/fad.) produced significantly the maximum fresh weight /plant at the two cuts in both seasons and their combined, except at $2^{\text {nd }}$ cut in the $1^{\text {st }}$ and $2^{\text {nd }}$ seasons, where the differences among planting densities did not reach the level of significance. 
Table 1. Fresh weight /plant (g) of sorghum as affected by forage sorghum genotypes, planting density and nitrogen fertilizer levels in the two seasons (2013 and 2014) and their combined

\begin{tabular}{|c|c|c|c|c|c|c|}
\hline \multirow[t]{2}{*}{ Main effects and interactions } & \multicolumn{2}{|c|}{ First season } & \multicolumn{2}{|c|}{ Second season } & \multicolumn{2}{|c|}{ Combined } \\
\hline & $1^{\text {st }}$ cut & $2^{\text {nd }}$ cut & $1^{\text {st }}$ cut & $2^{\text {nd }}$ cut & $1^{\text {st }}$ cut & $2^{\text {nd }}$ cut \\
\hline \multicolumn{7}{|l|}{ Forage sorghum genotype (G) } \\
\hline Sudan grass (Giza2) & $749.19 \mathrm{a}$ & $329.89 \mathrm{a}$ & $733.61 \mathrm{a}$ & $322.78 \mathrm{a}$ & $741.40 \mathrm{a}$ & $326.33 \mathrm{a}$ \\
\hline Sweet sorghum (Giza1) & $212.85 \mathrm{c}$ & $172.89 \mathrm{~b}$ & $210.28 \mathrm{c}$ & $170.83 \mathrm{~b}$ & $211.56 \mathrm{c}$ & $171.86 \mathrm{~b}$ \\
\hline Inter species Hybrid sorghum (Sx17) & $238.99 \mathrm{~b}$ & $147.92 \mathrm{c}$ & $236.67 \mathrm{~b}$ & $141.94 \mathrm{c}$ & $273.83 \mathrm{~b}$ & $144.93 \mathrm{c}$ \\
\hline F. test & $* *$ & $* *$ & $* *$ & $* *$ & $* *$ & $* *$ \\
\hline \multicolumn{7}{|l|}{ Planting density (D) } \\
\hline 70000 plants/ fad. & $411.20 \mathrm{a}$ & 220.93 & $404.07 \mathrm{a}$ & 218.15 & $407.64 \mathrm{a}$ & $219.54 \mathrm{a}$ \\
\hline 105000 plants/ fad. & $404.63 \mathrm{a}$ & 219.48 & $397.41 \mathrm{~b}$ & 214.07 & $401.02 \mathrm{ab}$ & $216.78 \mathrm{a}$ \\
\hline 140000 plants/ fad. & $401.19 \mathrm{a}$ & 214.59 & $395.19 \mathrm{c}$ & 207.04 & $398.19 \mathrm{~b}$ & $210.82 \mathrm{a}$ \\
\hline 210000 plants/ fad. & $384.36 \mathrm{~b}$ & 212.59 & $377.41 \mathrm{~d}$ & 208.15 & $380.89 \mathrm{c}$ & $210.37 b$ \\
\hline F. test & $* *$ & NS & $* *$ & NS & $* *$ & $* *$ \\
\hline \multicolumn{7}{|l|}{ Nitrogen fertilizer level (N) } \\
\hline $15 \mathrm{~kg} \mathrm{~N} / \mathrm{fad} . /$ cut & $353.57 \mathrm{c}$ & $191.58 \mathrm{c}$ & $348.06 \mathrm{c}$ & $187.5 \mathrm{c}$ & $350.81 \mathrm{c}$ & $189.54 \mathrm{c}$ \\
\hline $30 \mathrm{~kg} \mathrm{~N} /$ fad. / cut & $392.98 b$ & $213.31 \mathrm{~b}$ & $385.83 \mathrm{~b}$ & $210.28 \mathrm{~b}$ & $389.41 \mathrm{~b}$ & $211.79 \mathrm{~b}$ \\
\hline $45 \mathrm{~kg} \mathrm{~N} / \mathrm{fad} . /$ cut & $454.49 \mathrm{a}$ & $245.81 \mathrm{a}$ & $446.67 \mathrm{a}$ & $237.78 \mathrm{a}$ & $450.58 \mathrm{a}$ & $241.79 \mathrm{a}$ \\
\hline F. test & $* *$ & $* *$ & $* *$ & $* *$ & $* *$ & $* *$ \\
\hline \multicolumn{7}{|l|}{ Interactions } \\
\hline G $x$ D & $* *$ & NS & NS & NS & $* *$ & NS \\
\hline $\mathbf{G} \times \mathbf{N}$ & $* *$ & NS & $* *$ & NS & $* *$ & $*$ \\
\hline$D \times N$ & NS & NS & NS & NS & $*$ & NS \\
\hline
\end{tabular}

*,** and NS indicate significancy at 0.05 and 0.01 levels and insignificancy of differences, in respective order.

Table 2. Fresh forage yield/fad., (ton) of sorghum as affected by forage sorghum genotypes, plant density and nitrogen fertilizer levels in the two seasons (2013 and 2014) and their combined

\begin{tabular}{|c|c|c|c|c|c|c|}
\hline \multirow{2}{*}{ Main effects and interactions } & \multicolumn{2}{|c|}{ First season } & \multicolumn{2}{|c|}{ Second season } & \multicolumn{2}{|c|}{ Combined } \\
\hline & $1^{\text {st }}$ cut & $2^{\text {nd }}$ cut & $1^{\text {st }}$ cut & $2^{\text {nd }}$ cut & $1^{\text {st }}$ cut & $2^{\text {nd }}$ cut \\
\hline \multicolumn{7}{|l|}{ Forage sorghum genotype (G) } \\
\hline Sudan grass (Giza2) & $38.24 \mathrm{a}$ & $18.37 \mathrm{~b}$ & $36.07 \mathrm{a}$ & $17.09 \mathrm{~b}$ & $37.15 \mathrm{a}$ & $17.73 \mathrm{~b}$ \\
\hline Sweet sorghum (Giza1) & $19.93 \mathrm{c}$ & $22.10 \mathrm{a}$ & $17.82 \mathrm{c}$ & $20.75 \mathrm{a}$ & $18.88 \mathrm{c}$ & $21.43 \mathrm{a}$ \\
\hline Inter species Hybrid sorghum (Sx17) & $25.83 \mathrm{~b}$ & $16.16 \mathrm{c}$ & $23.69 \mathrm{~b}$ & $15.45 \mathrm{~b}$ & $24.76 \mathrm{~b}$ & $15.81 \mathrm{c}$ \\
\hline F.test & $* *$ & $* *$ & $* *$ & $* *$ & $* *$ & $* *$ \\
\hline \multicolumn{7}{|l|}{ Planting density (D) } \\
\hline 70000 plants/ fad. & $27.19 \mathrm{~b}$ & $18.58 \mathrm{~b}$ & $24.97 \mathrm{~b}$ & $17.55 \mathrm{~b}$ & $26.08 \mathrm{~b}$ & $18.06 \mathrm{~b}$ \\
\hline 105000 plants/ fad. & $27.15 b$ & $19.59 \mathrm{a}$ & $24.94 \mathrm{~b}$ & $18.38 \mathrm{a}$ & $26.04 \mathrm{~b}$ & $18.98 \mathrm{a}$ \\
\hline 140000 plants/ fad. & $29.08 \mathrm{a}$ & $19.65 \mathrm{a}$ & $26.58 \mathrm{a}$ & $18.60 \mathrm{a}$ & $27.83 \mathrm{a}$ & $19.12 \mathrm{a}$ \\
\hline 210000 plants/ fad. & $28.59 \mathrm{a}$ & $17.68 \mathrm{c}$ & $26.95 \mathrm{a}$ & $16.53 \mathrm{c}$ & $27.77 \mathrm{a}$ & $17.11 \mathrm{c}$ \\
\hline F. test & $* *$ & $* *$ & $* *$ & $* *$ & $* *$ & $* *$ \\
\hline \multicolumn{7}{|l|}{ Nitrogen fertilizer level (N) } \\
\hline $15 \mathrm{~kg} \mathrm{~N} / \mathrm{fad}$. / cut & $23.74 \mathrm{c}$ & $16.26 \mathrm{c}$ & $21.59 \mathrm{c}$ & $15.30 \mathrm{c}$ & $22.66 \mathrm{c}$ & $15.78 \mathrm{c}$ \\
\hline $30 \mathrm{~kg}$ N/ fad. / cut & $28.01 \mathrm{~b}$ & $18.74 \mathrm{~b}$ & $25.95 \mathrm{~b}$ & $17.70 \mathrm{~b}$ & $26.98 \mathrm{~b}$ & $18.22 \mathrm{~b}$ \\
\hline $45 \mathrm{~kg} \mathrm{~N} / \mathrm{fad} . /$ cut & $32.26 \mathrm{a}$ & $21.63 \mathrm{a}$ & $30.04 \mathrm{a}$ & $20.29 \mathrm{a}$ & $31.15 \mathrm{a}$ & $20.96 \mathrm{a}$ \\
\hline F. test & $* *$ & $* *$ & $* *$ & $* *$ & $* *$ & $* *$ \\
\hline \multicolumn{7}{|l|}{ Interactions } \\
\hline G $x D$ & NS & NS & $* *$ & $* *$ & $* *$ & $* *$ \\
\hline $\mathbf{G} \times \mathbf{N}$ & $* *$ & NS & $* *$ & NS & $* *$ & NS \\
\hline $\mathbf{D} \times \mathbf{N}$ & NS & NS & NS & NS & NS & $* *$ \\
\hline
\end{tabular}

*,** and NS indicate significancy at 0.05 and 0.01 levels and insignificancy of differences, in respective order. 


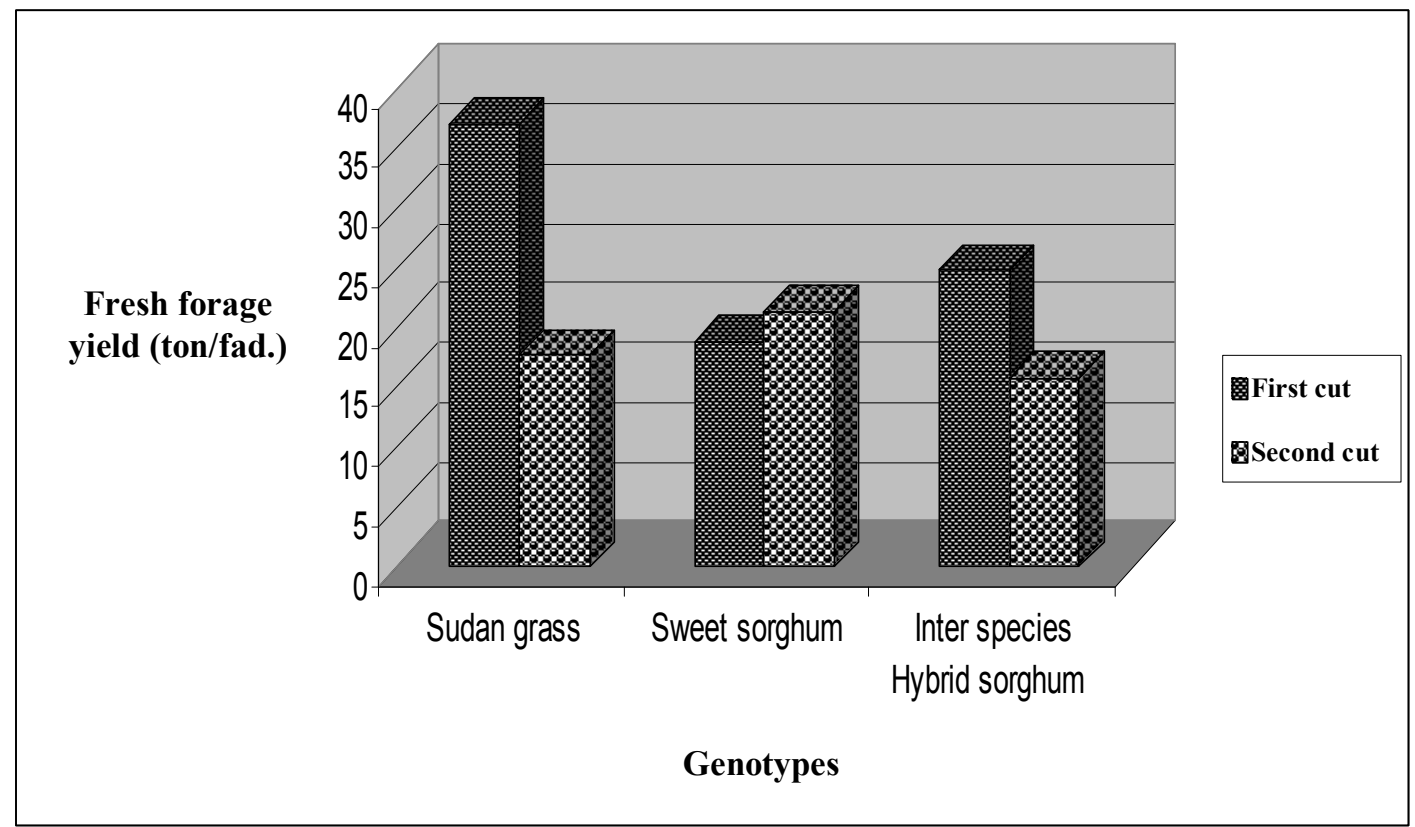

Fig. 1. Fresh forage yield (ton/fad.) of sorghum as affected by forage sorghum genotypes

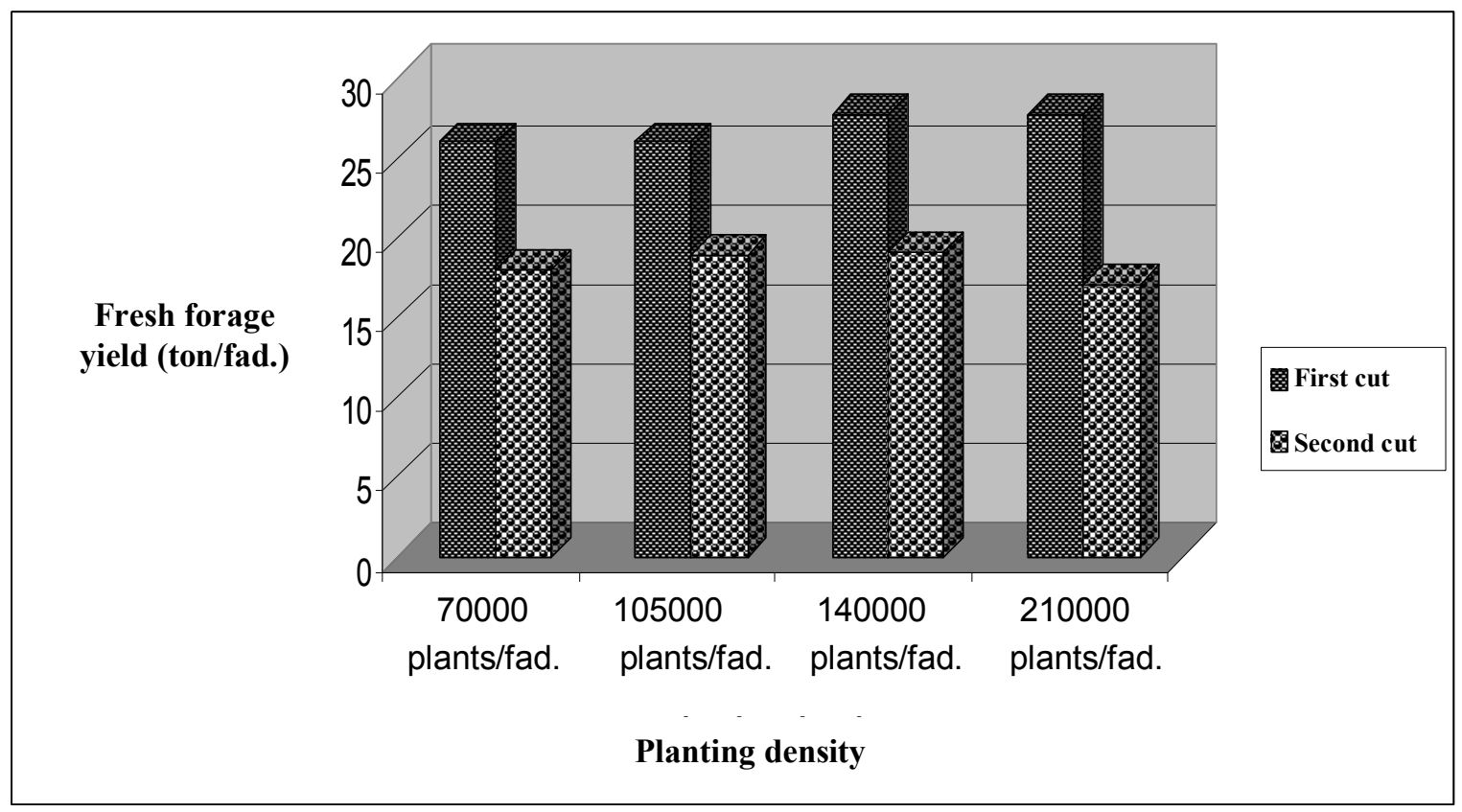

Fig. 2. Fresh forage yield (ton/ fad.) of sorghum as affected by planting density 


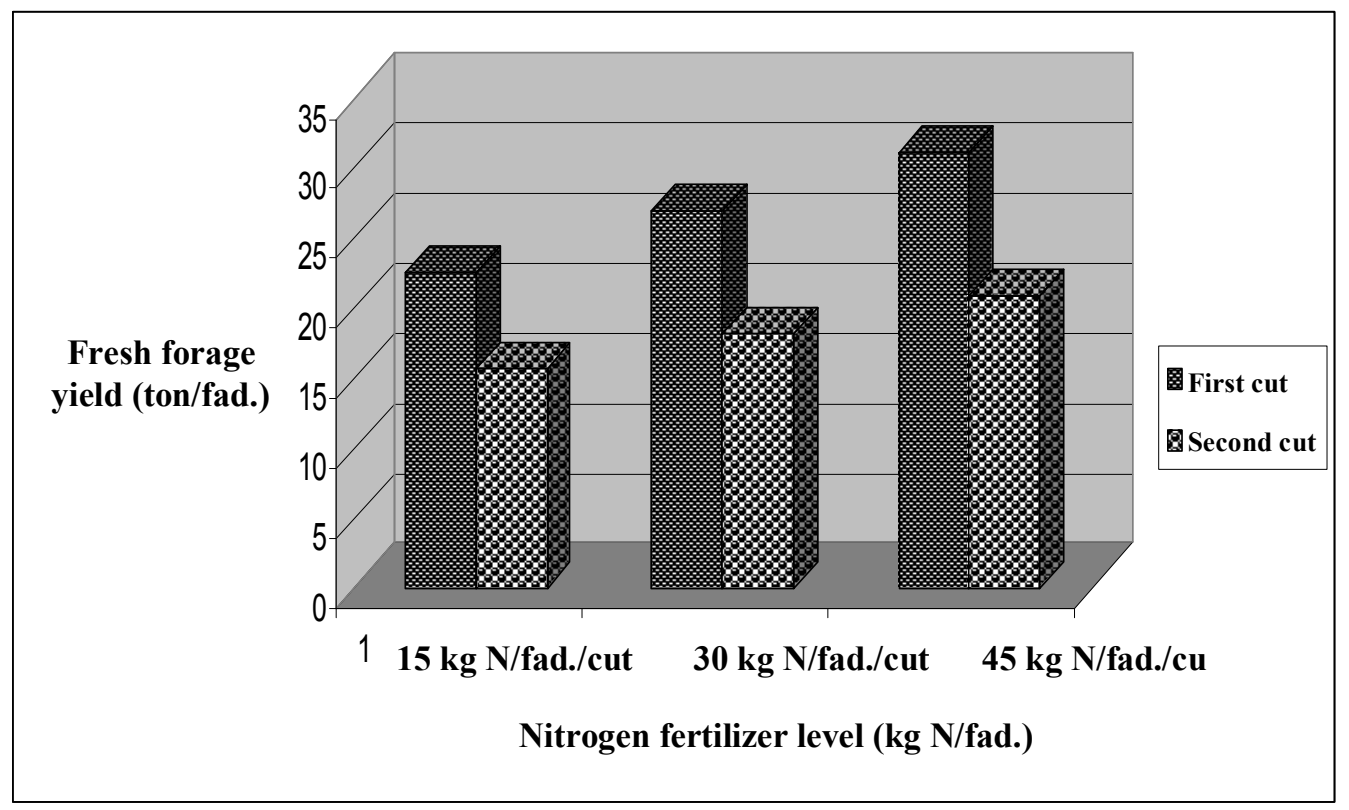

Fig. 3. Fresh forage yield (ton/ fad.) of sorghum as affected by $\mathbf{N}$ fertilizer level

On the other hand, 140000 plants/fad., produced the highest value of fresh forage yield./fad., and the difference did not reach the level of significant compared with the planting density 210000 plants/fad., (Table 2). It could be concluded that the dense plants suffered from competition for light, water and nutrient absorption. These results are in a good connection with those reported by El-Naggar (1983), Abd-Alla (1994), Mahmoud (1997), Bahrani and Deghani (2004), Turgut et al. (2005), Afzal et al. (2012), Mahmood et al. (2013) and Soleymani and Shahrajabian (2013).

\section{Nitrogen fertilizer levels effect}

It is evident from results (Tables 1 and 2) that increasing $\mathrm{N}$ level, resulted in a significant increase in fresh weight /plant and fresh forage yield/fad., where, the maximum value of aforementioned traits were obtained at the highest $\mathrm{N}$ level (45 $\mathrm{kg} \mathrm{N} /$ fad./cut) at $1^{\text {st }}$ and $2^{\text {nd }}$ cut in both seasons and their combined analysis. The average increase for the mean of both seasons for $1^{\text {st }}$ and $2^{\text {nd }} \mathrm{N}$ - increment reached about 11.0 and $28.4 \%$ for fresh weight ( $\mathrm{g} / \mathrm{plant}$ ), and 19.1 and $37.5 \%$ for fresh forage yield (ton/ fad.) at $1^{\text {st }}$ cut and 11.7 and $27.6 \%$ for fresh weight $(\mathrm{g} / \mathrm{plant})$ and 15.5 and $32.8 \%$ for fresh forage yield (ton/fad.) at $2^{\text {nd }}$ cut, respectively, in the combined analysis. According to these results, forage sorghum genotypes plants were in need for $\mathrm{N}$ fertilization. However, the aforementioned traits were highly responsed to this addition, where each $\mathrm{N}$ increment was accompanied by a significant increase in aforementioned traits. These results are interesting as they clearly indicate the positive and enhancing effect of nitrogen on the yield of forage sorghum plants. These results are in accordance with those found by Bahrani and Deghani (2004), Turgut et al. (2005), Afzal et al. (2012), Soleymani and Shahrajabian (2013), Abou-Amer and Kewan (2014) and Elshafey (2015).

\section{Interaction effect}

The interaction effect between forage sorghum genotypes $(G)$ and planting density (D) was highly significant on fresh weight /plant and fresh forage yield / fad., at $1^{\text {st }}$ cut in the combined data. Results in Tables $1-\mathrm{a}$ and 2-a, at the $1^{\text {st }}$ cut show that, the maximum value of aforementioned traits $(761.78 \mathrm{~g} /$ plant, $39.37 \mathrm{ton} /$ fad.) were recorded by planting sudan grass with 70000 plants/fad., for fresh weight/plant and at 210000 plants/fad., for fresh forage yield /fad., respectively, while the minimum values of aforementioned traits $(200.93 \mathrm{~g} / \mathrm{plant}, 18.47 \mathrm{ton} /$ fad.) were produced by planting sweet sorghum at planting density $210000 \mathrm{plants} / \mathrm{fad}$. for fresh weight/plant and at 70000 plants/fad., for fresh forage yield /fad. 
Table 1-a. Total plant fresh weight (g) of forage sorghum as influenced by the interaction between forage sorghum genotypes (G) and planting density (D) at $1^{\text {st }}$ cut (combined data)

\begin{tabular}{lcccc}
\hline Forage sorghum genotype (G) & \multicolumn{4}{c}{ Planting density (plant/fad.) } \\
\cline { 2 - 5 } & $\mathbf{7 0 0 0 0}$ & $\mathbf{1 0 5 0 0 0}$ & $\mathbf{1 4 0 0 0 0}$ & $\mathbf{2 1 0 0 0 0}$ \\
\hline \multirow{2}{*}{ Sudan grass } & $\mathrm{A}$ & $\mathrm{A}$ & $\mathrm{A}$ & $\mathrm{B}$ \\
& $761.78 \mathrm{a}$ & $751.22 \mathrm{a}$ & $739.08 \mathrm{a}$ & $713.53 \mathrm{a}$ \\
Sweet sorghum & $\mathrm{A}$ & $\mathrm{A}$ & $\mathrm{A}$ & $\mathrm{B}$ \\
& $218.92 \mathrm{c}$ & $217.69 \mathrm{c}$ & $208.71 \mathrm{c}$ & $200.93 \mathrm{c}$ \\
Interspecies hybrid sorghum & $\mathrm{A}$ & $\mathrm{AB}$ & $\mathrm{A}$ & $\mathrm{B}$ \\
& $242.21 \mathrm{~b}$ & $234.14 \mathrm{~b}$ & $246.78 \mathrm{~b}$ & $228.19 \mathrm{~b}$ \\
\hline
\end{tabular}

Table 2-a. Fresh forage yield (ton/fad.) of sorghum as influenced by the interaction between forage sorghum genotypes (G) and planting density (D) at $1^{\text {st }}$ cut (combined data)

\begin{tabular}{lcccc}
\hline Forage sorghum genotype (G) & \multicolumn{4}{c}{ Planting density (plant/fad.) } \\
\cline { 2 - 5 } & $\mathbf{7 0 0 0 0}$ & $\mathbf{1 0 5 0 0 0}$ & $\mathbf{1 4 0 0 0 0}$ & $\mathbf{2 1 0 0 0 0}$ \\
\hline \multirow{2}{*}{ Sudan grass } & $\mathrm{C}$ & $\mathrm{C}$ & $\mathrm{B}$ & $\mathrm{A}$ \\
& $35.27 \mathrm{a}$ & $35.20 \mathrm{a}$ & $38.78 \mathrm{a}$ & $39.37 \mathrm{a}$ \\
Sweet sorghum & $\mathrm{A}$ & $\mathrm{A}$ & $\mathrm{A}$ & $\mathrm{A}$ \\
& $18.47 \mathrm{c}$ & $18.52 \mathrm{c}$ & $19.42 \mathrm{c}$ & $19.10 \mathrm{c}$ \\
Interspecies hybrid sorghum & $\mathrm{A}$ & $\mathrm{A}$ & $\mathrm{A}$ & $\mathrm{A}$ \\
& $24.50 \mathrm{~b}$ & $24.40 \mathrm{~b}$ & $25.30 \mathrm{~b}$ & $24.85 \mathrm{~b}$ \\
\hline
\end{tabular}

The interaction effect between forage sorghum genotypes $(\mathrm{G})$ and nitrogen fertilizer levels $(\mathrm{N})$ was highly significant on fresh weight /plant and fresh forage yield /fad., at $1^{\text {st }}$ cut, in the combined data. Data in Tables (1-b and 2-b at the $1^{\text {st }}$ cut) show that, the maximum value of aforementioned traits $(827.06 \mathrm{~g} /$ plant, 42.18 ton/fad.) were produced by planting of sudan grass and adding $45 \mathrm{~kg} \mathrm{~N} /$ fad./cut., while, the minimum value (180.14 g/plant and $16.44 /$ fad.) were recorded by planting sweet sorghum under $15 \mathrm{~kg} \mathrm{~N} /$ fad./cut.

The significant interaction effect between planting density (D) and nitrogen fertilizer levels $(\mathrm{N})$ on fresh weight/plant (combined data, Table $1-\mathrm{c}$ at $1^{\text {st }}$ cut) indicate that the maximum value of fresh weight (459.03 g/plant) was produced at lowest planting density $(700000$ plants/fad.) under $45 \mathrm{~kg} \mathrm{~N} /$ fad./cut., while the minimum value of fresh weight $(329.73 \mathrm{~g} /$ plant $)$ was obtained at 210000 plants/ fad., under $15 \mathrm{~kg}$ $\mathrm{N} /$ fad./cut.

\section{Crude Protein (CP\%) and Crude Fiber Contents $(\mathrm{CF} \%)$ in Leaves and Sstems}

Results in Tables 3, 4, 5 and 6 show crude protein content $(\mathrm{CP} \%)$ and crude fiber contents $(\mathrm{CF} \%)$ in leaves and stems of three forage sorghum genotypes as affected by planting density and $\mathrm{N}$ fertilizer levels and their interactions at $1^{\text {st }}$ and $2^{\text {nd }}$ cut in the two seasons and their combined analysis. 
Table 1-b. Total plant fresh weight (g) of forage sorghum as influenced by the interaction between forage sorghum genotypes (G) and nitrogen fertilizer levels ( $\mathrm{kg} \mathrm{N} /$ fad./cut) at $1^{\text {st }}$ cut (combined data)

\begin{tabular}{lccc}
\hline \multirow{2}{*}{ Forage sorghum genotypes $(G)$} & \multicolumn{3}{c}{ Nitrogen fertilizer levels(kg N/fad./cut) } \\
\cline { 2 - 4 } & $\mathbf{1 5}$ & $\mathbf{3 0}$ & $\mathbf{4 5}$ \\
\hline \multirow{2}{*}{ Sudan grass } & $\mathrm{C}$ & $\mathrm{B}$ & $\mathrm{A}$ \\
& $670.49 \mathrm{a}$ & $726.66 \mathrm{a}$ & $827.06 \mathrm{a}$ \\
Sweet sorghum & $\mathrm{C}$ & $\mathrm{B}$ & $\mathrm{A}$ \\
& $180.14 \mathrm{c}$ & $208.08 \mathrm{c}$ & $246.48 \mathrm{c}$ \\
Interspecies hybrid sorghum & $\mathrm{C}$ & $\mathrm{B}$ & $\mathrm{A}$ \\
\hline
\end{tabular}

Table 2-b. Fresh forage yield (ton/fad.) of sorghum plants as influenced by the interaction between forage sorghum genotypes (G) and nitrogen fertilizer levels (kg N/fad./cut) at $1^{\text {st }}$ cut (combined data)

\begin{tabular}{lccc}
\hline Forage sorghum genotypes $(G)$ & \multicolumn{3}{c}{ Nitrogen fertilizer levels(kg N/fad./cut) } \\
\cline { 2 - 4 } & $\mathbf{1 5}$ & $\mathbf{3 0}$ & $\mathbf{4 5}$ \\
\hline \multirow{2}{*}{ Sudan grass } & $\mathrm{C}$ & $\mathrm{B}$ & $\mathrm{A}$ \\
& $31.72 \mathrm{a}$ & $37.56 \mathrm{a}$ & $42.18 \mathrm{a}$ \\
Sweet sorghum & $\mathrm{C}$ & $\mathrm{B}$ & $\mathrm{A}$ \\
& $16.44 \mathrm{c}$ & $18.40 \mathrm{c}$ & $21.80 \mathrm{c}$ \\
Interspecies hybrid sorghum & $\mathrm{C}$ & $\mathrm{B}$ & $\mathrm{A}$ \\
& $19.83 \mathrm{~b}$ & $24.98 \mathrm{~b}$ & $29.48 \mathrm{~b}$ \\
\hline
\end{tabular}

Table 1-c. Total plant fresh weight (g) of forage sorghum as influenced by the interaction between planting density (D) and nitrogen fertilizer levels $(\mathrm{kg} \mathrm{N} / \mathrm{fad} . / \mathrm{cut})$ at $1^{\text {st }}$ cut (combined data)

\begin{tabular}{lccc}
\hline Planting density (plant/fad.) & \multicolumn{4}{c}{ Nitrogen fertilizer levels(kg N/fad./cut) } \\
\cline { 2 - 4 } & $\mathbf{1 5}$ & $\mathbf{3 0}$ & $\mathbf{4 5}$ \\
\hline \multirow{2}{*}{$\mathbf{7 0 0 0 0}$} & $\mathrm{C}$ & $\mathrm{B}$ & $\mathrm{A}$ \\
& $360.00 \mathrm{a}$ & $403.88 \mathrm{a}$ & $459.03 \mathrm{a}$ \\
$\mathbf{1 0 5 0 0 0}$ & $\mathrm{C}$ & $\mathrm{B}$ & $\mathrm{A}$ \\
& $361.49 \mathrm{a}$ & $396.81 \mathrm{a}$ & $444.76 \mathrm{ab}$ \\
$\mathbf{1 4 0 0 0 0}$ & $\mathrm{C}$ & $\mathrm{B}$ & $\mathrm{A}$ \\
& $352.03 \mathrm{a}$ & $385.39 \mathrm{a}$ & $457.14 \mathrm{ab}$ \\
$\mathbf{2 1 0 0 0 0}$ & $\mathrm{C}$ & $\mathrm{B}$ & $\mathrm{A}$ \\
& $329.73 \mathrm{~b}$ & $371.54 \mathrm{~b}$ & $441.38 \mathrm{~b}$ \\
\hline
\end{tabular}


Table 3. Crude protein content (CP\%) in leaves of sorghum as affected by forage sorghum genotypes, planting density and nitrogen fertilizer levels in the two seasons (2013 and 2014) and their combined

\begin{tabular}{|c|c|c|c|c|c|c|}
\hline \multirow[t]{2}{*}{ Main effects and interactions } & \multicolumn{2}{|c|}{ First season } & \multicolumn{2}{|c|}{ Second season } & \multicolumn{2}{|c|}{ Combined } \\
\hline & $1^{\text {st }}$ cut & $2^{\text {nd }}$ cut & $1^{\text {st }}$ cut & $2^{\text {nd }}$ cut & $1^{\text {st }}$ cut & $2^{\text {nd }}$ cut \\
\hline \multicolumn{7}{|l|}{ Forage sorghum genotypes (G) } \\
\hline Sudan grass (Giza 2) & $13.34 b$ & $12.98 b$ & $13.18 \mathrm{~b}$ & $12.86 \mathrm{~b}$ & $13.26 \mathrm{~b}$ & $12.92 \mathrm{~b}$ \\
\hline Sweet sorghum (Giza 1) & $14.71 \mathrm{a}$ & $13.98 \mathrm{a}$ & $14.56 \mathrm{a}$ & $13.85 \mathrm{a}$ & $14.64 \mathrm{a}$ & $13.92 \mathrm{a}$ \\
\hline Inter species Hybrid sorghum (S x 17) & $12.61 \mathrm{c}$ & $11.52 \mathrm{c}$ & $12.46 \mathrm{c}$ & $11.40 \mathrm{c}$ & $12.53 \mathrm{c}$ & $11.46 \mathrm{c}$ \\
\hline F. test & $* *$ & $* *$ & $* *$ & $* *$ & $* *$ & $* *$ \\
\hline \multicolumn{7}{|l|}{ Planting density (D) } \\
\hline 70000 plants/ fad. & $13.92 \mathrm{a}$ & $13.26 \mathrm{a}$ & $13.77 \mathrm{a}$ & $13.11 \mathrm{a}$ & $13.85 \mathrm{a}$ & $13.19 \mathrm{a}$ \\
\hline 105000 plants/ fad. & $13.69 b$ & $12.97 \mathrm{~b}$ & $13.52 \mathrm{~b}$ & $12.81 \mathrm{~b}$ & $13.60 \mathrm{~b}$ & $12.89 \mathrm{~b}$ \\
\hline 140000 plants/ fad. & $13.46 \mathrm{c}$ & $12.63 \mathrm{c}$ & $13.29 \mathrm{c}$ & $12.51 \mathrm{c}$ & $13.38 \mathrm{c}$ & $12.57 \mathrm{c}$ \\
\hline 210000 plants/ fad. & $13.15 b$ & $12.46 \mathrm{~d}$ & $13.01 \mathrm{~d}$ & $12.38 \mathrm{~d}$ & $13.08 \mathrm{~d}$ & $12.42 \mathrm{~d}$ \\
\hline F. test & $* *$ & $* *$ & $* *$ & $* *$ & $* *$ & $* *$ \\
\hline \multicolumn{7}{|l|}{ Nitrogen fertilizer level (N) } \\
\hline $15 \mathrm{~kg} \mathrm{~N} /$ fad. / cut & $13.24 \mathrm{c}$ & $12.36 \mathrm{c}$ & $13.09 \mathrm{c}$ & $12.22 \mathrm{c}$ & $13.17 \mathrm{c}$ & $12.29 \mathrm{c}$ \\
\hline $30 \mathrm{~kg} \mathrm{~N} /$ fad. / cut & $13.55 b$ & $12.86 \mathrm{~b}$ & $13.42 \mathrm{~b}$ & $12.73 \mathrm{~b}$ & $13.49 \mathrm{~b}$ & $12.80 \mathrm{~b}$ \\
\hline $45 \mathrm{~kg} \mathrm{~N} /$ fad. / cut & $13.87 \mathrm{a}$ & $13.26 \mathrm{a}$ & $13.68 \mathrm{a}$ & $13.16 \mathrm{a}$ & $13.78 \mathrm{a}$ & $13.21 \mathrm{a}$ \\
\hline F. test & $* *$ & $* *$ & $* *$ & $* *$ & $* *$ & $* *$ \\
\hline \multicolumn{7}{|l|}{ Interactions } \\
\hline G $\times D$ & NS & $* *$ & NS & $* *$ & NS & $* *$ \\
\hline $\mathbf{G} \times \mathbf{N}$ & $*$ & $* *$ & NS & NS & NS & $* *$ \\
\hline D $x \mathbf{N}$ & NS & NS & NS & NS & NS & $* *$ \\
\hline
\end{tabular}

*** and NS indicate significancy at 0.05 and 0.01 levels and insignificancy of differences, in respective order.

Table 4. Crude protein content $(\mathrm{CP} \%)$ in stems of sorghum as affected by forage sorghum genotypes, planting density and nitrogen fertilizer levels in the two seasons (2013 and 2014) and their combined

\begin{tabular}{|c|c|c|c|c|c|c|}
\hline \multirow[t]{2}{*}{ Main effects and interactions } & \multicolumn{2}{|c|}{ First season } & \multicolumn{2}{|c|}{ Second season } & \multicolumn{2}{|c|}{ Combined } \\
\hline & $1^{\text {st }}$ cut & $2^{\text {nd }}$ cut & $1^{\text {st }}$ cut & $2^{\text {nd }}$ cut & $1^{\text {st }}$ cut & $2^{\text {nd }}$ cut \\
\hline \multicolumn{7}{|l|}{$\overline{\text { Forage sorghum genotypes (G) }}$} \\
\hline Sudan grass (Giza2) & $12.75 \mathrm{~b}$ & $12.19 \mathrm{a}$ & $12.57 \mathrm{~b}$ & $12.08 \mathrm{a}$ & $12.66 \mathrm{~b}$ & $12.14 \mathrm{a}$ \\
\hline Sweet sorghum (Giza1) & $13.59 \mathrm{a}$ & $12.17 \mathrm{a}$ & $13.41 \mathrm{a}$ & $12.07 \mathrm{a}$ & $13.50 \mathrm{a}$ & $12.12 \mathrm{a}$ \\
\hline Inter species Hybrid sorghum (Sx17) & $11.06 \mathrm{c}$ & $9.88 b$ & $10.90 \mathrm{c}$ & $9.82 \mathrm{~b}$ & $10.98 \mathrm{c}$ & $9.85 \mathrm{~b}$ \\
\hline F. test & $* *$ & $* *$ & $* *$ & $* *$ & $* *$ & $* *$ \\
\hline \multicolumn{7}{|l|}{ Planting density (D) } \\
\hline 70000 plants/ fad. & $12.92 \mathrm{a}$ & $12.20 \mathrm{a}$ & $12.72 \mathrm{a}$ & $11.89 \mathrm{a}$ & $12.82 \mathrm{a}$ & $12.04 \mathrm{a}$ \\
\hline 105000 plants/ fad. & $12.66 b$ & $11.68 b$ & $12.46 \mathrm{~b}$ & $11.63 \mathrm{~b}$ & $12.56 \mathrm{~b}$ & $11.65 \mathrm{~b}$ \\
\hline 140000 plants/ fad. & $12.36 \mathrm{c}$ & $11.25 \mathrm{c}$ & $12.19 \mathrm{c}$ & $11.22 \mathrm{c}$ & $12.27 \mathrm{c}$ & $11.23 \mathrm{c}$ \\
\hline 210000 plants/ fad. & $11.94 b$ & $10.53 d$ & $11.79 \mathrm{~d}$ & $10.57 \mathrm{~d}$ & $11.87 \mathrm{~d}$ & $10.55 \mathrm{~d}$ \\
\hline F. test & $* *$ & $* *$ & $* *$ & $* *$ & $* *$ & $* *$ \\
\hline \multicolumn{7}{|l|}{ Nitrogen fertilizer level (N) } \\
\hline $15 \mathrm{~kg} \mathrm{~N} / \mathrm{fad} . /$ cut & $11.96 \mathrm{c}$ & $10.97 \mathrm{c}$ & $11.76 \mathrm{c}$ & $10.97 \mathrm{c}$ & $11.86 \mathrm{c}$ & $10.97 \mathrm{c}$ \\
\hline $30 \mathrm{~kg} \mathrm{~N} / \mathrm{fad} . /$ cut & $12.48 b$ & $11.39 b$ & $12.33 \mathrm{~b}$ & $11.24 \mathrm{~b}$ & $12.40 \mathrm{~b}$ & $11.32 \mathrm{~b}$ \\
\hline $45 \mathrm{~kg} \mathrm{~N} / \mathrm{fad} . /$ cut & $12.96 a$ & $11.88 \mathrm{a}$ & $12.79 \mathrm{a}$ & $11.76 \mathrm{a}$ & $12.87 \mathrm{a}$ & $11.82 \mathrm{a}$ \\
\hline F. test & $* *$ & $* *$ & $* *$ & $* *$ & $* *$ & $* *$ \\
\hline \multicolumn{7}{|l|}{ Interactions } \\
\hline G $\times D$ & $* *$ & $* *$ & NS & NS & $* *$ & $* *$ \\
\hline $\mathbf{G} \times \mathbf{N}$ & $* *$ & NS & $*$ & NS & $* *$ & NS \\
\hline$D \times N$ & NS & $* *$ & NS & NS & $* *$ & $*$ \\
\hline
\end{tabular}

$*, * *$ and NS indicate significancy at 0.05 and 0.01 levels and insignificancy of differences, in respective order. 
Table 5. Crude fiber content (CF\%) in leaves of sorghum as affected by forage sorghum genotypes, planting density and nitrogen fertilizer levels in the two seasons (2013 and 2014) and their combined

\begin{tabular}{|c|c|c|c|c|c|c|}
\hline \multirow[t]{2}{*}{ Main effects and interactions } & \multicolumn{2}{|c|}{$\overline{\text { First season }}$} & \multicolumn{2}{|c|}{ Second season } & \multicolumn{2}{|c|}{ Combined } \\
\hline & $\mathbf{1}^{\text {st }}$ cut & $2^{\text {nd }}$ cut & $1^{\text {st }}$ cut & $2^{\text {nd }}$ cut & $1^{\text {st }}$ cut & $2^{\text {nd }}$ cut \\
\hline \multicolumn{7}{|l|}{ Forage sorghum genotypes (G) } \\
\hline Sudan grass (Giza 2) & $28.76 \mathrm{~b}$ & $28.65 \mathrm{~b}$ & $28.58 \mathrm{~b}$ & $28.52 \mathrm{~b}$ & $28.67 \mathrm{~b}$ & $28.59 \mathrm{~b}$ \\
\hline Sweet sorghum (Giza 1) & $31.80 \mathrm{a}$ & $32.35 \mathrm{a}$ & $31.33 \mathrm{a}$ & $32.15 \mathrm{a}$ & $31.56 \mathrm{a}$ & $32.25 \mathrm{a}$ \\
\hline Inter species Hybrid sorghum (S x 17) & $27.77 \mathrm{c}$ & $28.24 \mathrm{c}$ & $27.56 \mathrm{c}$ & $28.08 \mathrm{c}$ & $27.66 \mathrm{c}$ & $18.16 \mathrm{c}$ \\
\hline F. test & $* *$ & $* *$ & $* *$ & $* *$ & $* *$ & $* *$ \\
\hline \multicolumn{7}{|l|}{ Planting density (D) } \\
\hline 70000 plants/ fad. & $30.34 \mathrm{a}$ & $30.16 \mathrm{a}$ & $29.77 \mathrm{a}$ & $29.97 \mathrm{a}$ & $30.06 \mathrm{a}$ & $30.07 \mathrm{~b}$ \\
\hline 105000 plants/fad. & $29.82 b$ & $30.26 \mathrm{a}$ & $29.53 \mathrm{a}$ & $30.12 \mathrm{a}$ & $29.67 b$ & $30.19 \mathrm{a}$ \\
\hline 140000 plants/fad. & $29.24 \mathrm{c}$ & $29.74 b$ & $28.90 \mathrm{~b}$ & $29.60 \mathrm{~b}$ & $29.07 \mathrm{c}$ & $29.67 \mathrm{c}$ \\
\hline 210000 plants/fad. & $28.37 \mathrm{~d}$ & $28.83 \mathrm{c}$ & $28.41 \mathrm{~b}$ & $28.64 \mathrm{c}$ & $28.39 \mathrm{~d}$ & $28.74 \mathrm{~d}$ \\
\hline F. test & $* *$ & $* *$ & $* *$ & $* *$ & $* *$ & $* *$ \\
\hline \multicolumn{7}{|l|}{ Nitrogen fertilizer level $(\mathbf{N})$ : } \\
\hline $15 \mathrm{~kg} \mathrm{~N} /$ fad. $/ \mathrm{cut}$ & $30.08 \mathrm{a}$ & $30.61 \mathrm{a}$ & $29.48 \mathrm{a}$ & $30.45 \mathrm{a}$ & $29.78 \mathrm{a}$ & $30.53 \mathrm{a}$ \\
\hline $30 \mathrm{~kg} \mathrm{~N} /$ fad./cut & $29.52 \mathrm{~b}$ & $29.42 \mathrm{~b}$ & $29.20 \mathrm{a} \mathrm{b}$ & $29.24 \mathrm{~b}$ & $29.36 \mathrm{~b}$ & $29.33 \mathrm{~b}$ \\
\hline $45 \mathrm{~kg} \mathrm{~N} /$ fad./cut & $28.72 \mathrm{c}$ & $29.22 \mathrm{c}$ & $28.78 \mathrm{~b}$ & $29.05 \mathrm{c}$ & $28.75 \mathrm{c}$ & $29.13 \mathrm{c}$ \\
\hline F. test & $* *$ & $* *$ & $*$ & $* *$ & $* *$ & $* *$ \\
\hline \multicolumn{7}{|l|}{ Interactions } \\
\hline G $\times D$ & $* *$ & $* *$ & NS & $* *$ & $* *$ & $* *$ \\
\hline $\mathbf{G} \times \mathbf{N}$ & $* *$ & $* *$ & NS & $* *$ & $* *$ & $* *$ \\
\hline D $x \mathbf{N}$ & $* *$ & $* *$ & NS & $* *$ & $*$ & $* *$ \\
\hline
\end{tabular}

*** and NS indicate significancy at 0.05 and 0.01 levels and insignificancy of differences, in respective order.

Table 6. Crude fiber content (CF\%) in stems of sorghum as affected by forage sorghum genotypes, planting density and nitrogen fertilizer levels in the two seasons (2013 and 2014) and their combined

\begin{tabular}{|c|c|c|c|c|c|c|}
\hline \multirow[t]{2}{*}{ Main effects and interactions } & \multicolumn{2}{|c|}{ First season } & \multicolumn{2}{|c|}{ Second season } & \multicolumn{2}{|c|}{ Combined } \\
\hline & $1^{\text {st }}$ cut & $2^{\text {nd }}$ cut & $1^{\text {st }}$ cut & $2^{\text {nd }}$ cut & $1^{\text {st }}$ cut & $2^{\text {nd }}$ cut \\
\hline \multicolumn{7}{|l|}{ Forage sorghum genotypes (G) } \\
\hline Sudan grass(Giza2) & $28.37 \mathrm{~b}$ & $28.78 \mathrm{~b}$ & $28.16 \mathrm{a}$ & $28.33 \mathrm{a}$ & $28.26 \mathrm{a}$ & $28.55 \mathrm{~b}$ \\
\hline Sweet sorghum(Giza1) & $28.48 \mathrm{a}$ & $28.93 \mathrm{a}$ & $28.17 \mathrm{a}$ & $28.70 \mathrm{a}$ & $28.33 \mathrm{a}$ & $28.81 \mathrm{a}$ \\
\hline Inter species Hybrid sorghum (S x 17) & $26.44 \mathrm{c}$ & $26.98 \mathrm{c}$ & $26.22 \mathrm{~b}$ & $26.98 \mathrm{a}$ & $26.33 \mathrm{~b}$ & $26.98 \mathrm{c}$ \\
\hline F. test & $* *$ & $* *$ & $* *$ & $* *$ & $* *$ & $* *$ \\
\hline \multicolumn{7}{|l|}{ Planting density (D) } \\
\hline 70000 plants/ fad. & $28.31 \mathrm{a}$ & $28.71 \mathrm{a}$ & $28.05 \mathrm{a}$ & $28.14 \mathrm{ab}$ & $28.18 \mathrm{a}$ & $28.42 \mathrm{a}$ \\
\hline 105000 plants/ fad. & $28.02 \mathrm{~b}$ & $28.43 \mathrm{~b}$ & $27.73 \mathrm{~b}$ & $28.23 \mathrm{a}$ & $27.87 \mathrm{~b}$ & $28.33 \mathrm{a}$ \\
\hline 140000 plants/ fad. & $27.50 \mathrm{c}$ & $28.12 \mathrm{c}$ & $27.22 \mathrm{c}$ & $27.97 \mathrm{~b}$ & $27.36 \mathrm{c}$ & $28.05 \mathrm{~b}$ \\
\hline 210000 plants/ fad. & $27.23 \mathrm{~d}$ & $27.64 \mathrm{~d}$ & $27.06 \mathrm{c}$ & $27.68 \mathrm{c}$ & $27.14 \mathrm{~d}$ & $27.66 \mathrm{c}$ \\
\hline F. test & $* *$ & $* *$ & $* *$ & $* *$ & $* *$ & $* *$ \\
\hline \multicolumn{7}{|l|}{ Nitrogen fertilizer level (N) } \\
\hline $15 \mathrm{~kg}$ N/ fad. / cut & $28.21 \mathrm{a}$ & $28.75 \mathrm{a}$ & $28.02 \mathrm{a}$ & $28.44 \mathrm{a}$ & $28.12 \mathrm{a}$ & $28.60 \mathrm{a}$ \\
\hline $30 \mathrm{~kg}$ N/ fad. / cut & $27.71 \mathrm{~b}$ & $28.26 \mathrm{~b}$ & $27.36 \mathrm{~b}$ & $27.94 \mathrm{~b}$ & $27.54 \mathrm{~b}$ & $28.10 \mathrm{~b}$ \\
\hline $45 \mathrm{~kg} \mathrm{~N} / \mathrm{fad} . /$ cut & $27.36 \mathrm{c}$ & $27.66 \mathrm{c}$ & $27.17 \mathrm{c}$ & $27.63 \mathrm{~b}$ & $27.27 \mathrm{c}$ & $27.65 \mathrm{c}$ \\
\hline F. test & $* *$ & $* *$ & $* *$ & $* *$ & $* *$ & $* *$ \\
\hline \multicolumn{7}{|l|}{ Interactions } \\
\hline G $\times D$ & NS & $* *$ & NS & $* *$ & NS & $* *$ \\
\hline $\mathbf{G} \times \mathbf{N}$ & $* *$ & NS & NS & NS & NS & NS \\
\hline $\mathbf{D} \times \mathbf{N}$ & $* *$ & NS & NS & NS & $* *$ & NS \\
\hline
\end{tabular}




\section{Forage sorghum genotypes effect}

Crude protein and fiber percentage variations among the forage sorghum genotypes seemed to be significant at $1^{\text {st }}$ and $2^{\text {nd }}$ cuttings in both seasons and the combined analysis. These differences play a major role in determining not only a plant's ability to survive in the ecosystem, but also its potential productivity. Sweet sorghum gave higher value of crude protein and fiber contents in leaves and stems, sudan grass ranked the second and followed by interspecies hybrid sorghum at $1^{\text {st }}$ and $2^{\text {nd }}$ cut in the $1^{\text {st }}$ and $2^{\text {nd }}$ seasons as well as in the combined analysis. El-Naggar (1983), Almodares et al. (2009), Ayub et al. (2010), Mahmood et al. (2013) and Soleymani and Shahrajabian (2013) came to similar results on Sudanese sorghum.

\section{Planting density effect}

Data presented in Tables 3, 4, 5 and 6 show that crude protein content $(\mathrm{CP} \%)$ and crude fiber content (CF\%) in leaves and stems significantly decreased by increasing planting density from 70000 to 210000 plants/fad. The highest planting density gave the minimum protein and fiber percentages in leaves and stems at $1^{\text {st }}$ and $2^{\text {nd }}$ cuts in first, second seasons and their combined. Similar results were obtained by Geweifel (1990) in his investigation on fodder maize. Also, ElNaggar (1983), Abd-Alla (1994), Mahmoud (1997) and Bahrani and Deghani (2004) reported that increasing planting density decreased the percentage of protein and fiber in leaves, stalks and whole sorghum plant. While, Mahmood et al. (2013) found that planting density had no clear influence on most of the quality parameters, while Sanderson et al. (1995) found that increasing plant density increased fiber content in forage corn plants.

\section{Nitrogen fertilizer levels effect}

Results presented in Tables 3, 4, 5 and 6 show that crude protein content $(\mathrm{CP} \%)$ and crude fiber content $(\mathrm{CF} \%)$ in leaves and stems were significantly influenced by $\mathrm{N}$ - application . Where, raising nitrogen fertilizer level from 15 to 30 and up to $45 \mathrm{~kg} \mathrm{~N} /$ fad./cut led to a gradual increase in $\mathrm{CP}$, while $\mathrm{CF} \%$ showed a gradual decrease in $1^{\text {st }}$ and $2^{\text {nd }}$ cuttings in both seasons and their combined. These results are in harmony with those reported by Almodares et al. (2009), Mahmood et al. (2013), Soleymani and Shahrajabian (2013) and Abou-Amer and Kewan (2014).

\section{Interaction effect}

The interaction effect between forage sorghum genotypes (G) and planting density (D) was highly significant on crude protein and fiber contents, in the combined data. Data in Tables $3-\mathrm{a}, 4-\mathrm{a}$ and $5-\mathrm{a}$ at the $2^{\text {nd }}$ cut show that, the maximum $\mathrm{CP} \%$ and $\mathrm{CF} \%(14.05,13.91$ and $32.27 \%$ ) in leaves and stems, respectively were recorded by planting sweet sorghum at plant population density 70000 plants/fad.

The interaction effect between forage sorghum genotypes $(\mathrm{G})$ and nitrogen fertilizer levels $(\mathrm{N})$ was highly significant on $\mathrm{CP} \%$ in leaves at $2^{\text {nd }}$ cut, in the combined data. Data in Table 3-b show that, the maximum CP\% (14.33\%) was produced by planting of sweet sorghum plants and adding $45 \mathrm{~kg} \mathrm{~N} /$ fad./ cut. while, the minimum value of $\mathrm{CP}(11.00 \%)$ was recorded by planting interspecies hybrid sorghum plants under $15 \mathrm{~kg} \mathrm{~N} /$ fad./cut.

\section{Conclusion}

Results of this experiments revealed that increasing planting density up to 140000 plants/ fad. led to increase fresh and dry forage yield /fad., but fresh and dry weight /plant, CP \% and $\mathrm{CF} \%$ in leaves and stems were decreased. Application of $\mathrm{N}$ fertilizer up to $45 \mathrm{~kg} \mathrm{~N} /$ fad., significantly increased fresh weight /plant, fresh forage yield /fad., and CP \%, while CF \% was significantly decreased. 
Table 3-a. Crude protein (CP\%) in leaves of sorghum plants as influenced by the interaction between forage sorghum genotypes $(G)$ and planting density (D) at $2^{\text {nd }}$ cut (combined data)

\begin{tabular}{lcccc}
\hline Forage sorghum genotype (G) & \multicolumn{4}{c}{ Planting density (plant/fad.) } \\
\cline { 2 - 5 } & $\mathbf{7 0 0 0 0}$ & $\mathbf{1 0 5 0 0 0}$ & $\mathbf{1 4 0 0 0 0}$ & $\mathbf{2 1 0 0 0 0}$ \\
\hline \multirow{2}{*}{ Sudan grass } & $\mathrm{A}$ & $\mathrm{B}$ & $\mathrm{B}$ & $\mathrm{C}$ \\
& $13.40 \mathrm{~b}$ & 12.83 & $12.81 \mathrm{~b}$ & $12.64 \mathrm{~b}$ \\
Sweet sorghum & $\mathrm{A}$ & $\mathrm{A}$ & $\mathrm{B}$ & $\mathrm{B}$ \\
& $14.05 \mathrm{a}$ & $14.10 \mathrm{a}$ & $13.78 \mathrm{a}$ & 13.73 \\
Interspecies hybrid sorghum & $\mathrm{A}$ & $\mathrm{B}$ & $\mathrm{C}$ & $\mathrm{D}$ \\
& $12.11 \mathrm{c}$ & $11.74 \mathrm{c}$ & $11.11 \mathrm{c}$ & $10.89 \mathrm{c}$ \\
\hline
\end{tabular}

Table 3-b. Crude protein (CP\%) in leaves of sorghum plants as influenced by the interaction between forage sorghum genotypes $(G)$ and nitrogen fertilizer levels at $2^{\text {nd }}$ cut (combined data)

\begin{tabular}{lccc}
\hline Forage sorghum genotype $(G)$ & \multicolumn{3}{c}{ Nitrogen fertilizer level (kg N/fad./cut) } \\
\cline { 2 - 4 } & $\mathbf{1 5}$ & $\mathbf{3 0}$ & $\mathbf{4 5}$ \\
\hline \multirow{2}{*}{ Sudan grass } & $\mathrm{C}$ & $\mathrm{B}$ & $\mathrm{A}$ \\
& $12.53 \mathrm{~b}$ & $12.93 \mathrm{~b}$ & $13.30 \mathrm{~b}$ \\
Sweet sorghum & $\mathrm{C}$ & $\mathrm{B}$ & $\mathrm{A}$ \\
& $13.34 \mathrm{a}$ & $14.08 \mathrm{a}$ & $14.33 \mathrm{a}$ \\
Interspecies hybrid sorghum & $\mathrm{C}$ & $\mathrm{B}$ & $\mathrm{A}$ \\
& $11.00 \mathrm{c}$ & $11.38 \mathrm{c}$ & $12.01 \mathrm{c}$ \\
\hline
\end{tabular}

Table 4-a. Crude protein (CP\%) in stems of sorghum plants as influenced by the interaction between forage sorghum genotypes $(G)$ and planting density $(D)$ at $1^{\text {st }}$ cut (combined data)

\begin{tabular}{lcccc}
\hline Forage sorghum genotype (G) & \multicolumn{4}{c}{ Planting density (plant/fad.) } \\
\cline { 2 - 5 } & $\mathbf{7 0 0 0 0}$ & $\mathbf{1 0 5 0 0 0}$ & $\mathbf{1 4 0 0 0 0}$ & $\mathbf{2 1 0 0 0 0}$ \\
\hline \multirow{2}{*}{ Sudan grass } & $\mathrm{A}$ & $\mathrm{A}$ & $\mathrm{B}$ & $\mathrm{C}$ \\
& $12.97 \mathrm{~b}$ & $12.85 \mathrm{~b}$ & $12.64 \mathrm{~b}$ & $12.18 \mathrm{~b}$ \\
Sweet sorghum & $\mathrm{A}$ & $\mathrm{B}$ & $\mathrm{B}$ & $\mathrm{C}$ \\
& $13.91 \mathrm{a}$ & $13.65 \mathrm{a}$ & $13.52 \mathrm{a}$ & $12.92 \mathrm{a}$ \\
Interspecies hybrid sorghum & $\mathrm{A}$ & $\mathrm{B}$ & $\mathrm{C}$ & $\mathrm{C}$ \\
& $11.58 \mathrm{c}$ & $11.18 \mathrm{c}$ & $10.66 \mathrm{c}$ & $10.50 \mathrm{c}$ \\
\hline
\end{tabular}


Table 5-a. Crude fiber content $(\mathrm{CF} \%)$ in leaves of sorghum plants as influenced by the interaction between forage sorghum genotypes $(G)$ and planting density (D) at $1^{\text {st }}$ cut (combined data)

\begin{tabular}{lcccc}
\hline Forage sorghum genotype (G) & \multicolumn{4}{c}{ Planting density (plant/fad.) } \\
\cline { 2 - 5 } & $\mathbf{7 0 0 0 0}$ & $\mathbf{1 0 5 0 0 0}$ & $\mathbf{1 4 0 0 0 0}$ & $\mathbf{2 1 0 0 0 0}$ \\
\hline \multirow{2}{*}{ Sudan grass } & $\mathrm{A}$ & $\mathrm{B}$ & $\mathrm{B}$ & $\mathrm{B}$ \\
& $29.34 \mathrm{~b}$ & $28.84 \mathrm{~b}$ & $28.46 \mathrm{~b}$ & $28.04 \mathrm{~b}$ \\
Sweet sorghum & $\mathrm{A}$ & $\mathrm{A}$ & $\mathrm{B}$ & $\mathrm{C}$ \\
& $32.37 \mathrm{a}$ & $32.24 \mathrm{a}$ & $31.52 \mathrm{a}$ & $30.11 \mathrm{a}$ \\
Interspecies hybrid sorghum & $\mathrm{A}$ & $\mathrm{B}$ & $\mathrm{C}$ & $\mathrm{C}$ \\
& $28.47 \mathrm{c}$ & $27.93 \mathrm{c}$ & $27.24 \mathrm{c}$ & $27.01 \mathrm{c}$ \\
\hline
\end{tabular}

\section{REFERENCES}

AOAC (1990). Association of Official Agriculture Chemists "Official Methods of Analysis $15^{\text {th }}$ Ed. Washington, D.C.

Abd-Alla, S.O.M. (1994). Response of sweet sorghum to some agronomic treatments under Siwa Oasis conditions. MSc. Thesis, Agron. Dept., Fac. Agric., Ain Shams Univ., Egypt.

Abou-Amer, A.I. and K. Z. Kewan (2014). Effect of NP fertilization levels on sorghum (Sorghum bicolor L.) yield and fodder quality for animals. Alex. J. Agric. Res., 59 (1) : 5159.

Afzal, M., A. Ahmad and A.U.H. Ahmad (2012). Effect of nitrogen on growth and yield of sorghum forage (Sorghum bicolor L.) moench cv.) under three cuttings system. Cercetări Agronomice în Moldova, XLV (4): 152.

Almodares, A., M. Jafarinia and M.R. Hadi (2009). The effects of nitrogen fertilizer on chemical compositions in corn and sweet sorghum. American-Eurasian J. Agric. and Environ. Sci., 6 (4): 441-446.

Almodares, A., R. Taheri, M.R. Hadi and M. Fathi (2006). The effect of nitrogen and potassium fertilizers on the growth parameters and the yield components of two sweet sorghum cultivars. Pak. J. Biol. Sci., 9: 2350-2353.

Ayub, M., M.A. Nadeem, M.T. Abdul Ghafoor, Z. Ahmed and M. Naeem (2010). Comparative studies on the growth, forage yield and quality of sorghum (Sorghum Bicolor L.) varieties under irrigated conditions of Faisalabad. Pak. J. Llife Soc. Sci., 8 (2): 94-97.

Bahrani, M.J. and A. Deghani (2004). Summer forage sorghum yield, protein and prussic acid contents as affected by plant density and nitrogen topdressing. J. Agric. Sci. Technol., 6: 73-83.

Bozorgvar, N., M.M. Khademosharieh, E. Neamatollah and M.R. Jahansuz (2013). Determine the best varieties of forage sorghum. Advances in Environ. Biol., 7 (6): 1105-1112.

Duncan, B.D. (1955). Multiple Range and Multiple F test. Biometrics, 11: 1-42.

El-Naggar, H.M.M.M. (1983). Response of forage sorghum to some cultural treatments. MSc. Thesis, Agron. Dept., Fac. Agric., Moshtohor, Zagazig Univ., Egypt.

Elshafey, E.G. (2015). Nitrogen accumulation in some forage crops. M. Sc. Thesis, Agric. Sci. Fac. Agric., Cairo Univ., Egypt. 
Geweifel, H.G.M. (1990). Forage yield and quality of fodder maize as influenced by cutting date, seeding rate and nitrogen fertilization. Egypt. J. Appl. Sci., 5 (8): 832845.

Hussein, M.M. and M.M. Sabbour (2014). Effect of irrigation intervals and nitrogen fertilizer on yield and water use efficiency of sorghum fodder. Int. J. Sci. and Res., 3 (11): 404-410.

Mahmood, A., A.N. Habib Ullah, H. Shahzad, S. Ali, M. Ahmad, Z. Ul-Haq, B. Honermeier and M. Hasanuzzaman (2013). Dry matter yield and chemical composition of sorghum cultivars with varying planting density and sowing date. Sains Malaysiana, 42 (10): 1529-1538.

Mahmoud, T.A. (1997). Response of some forage crops to different seeding rates. 1Forage sorghum hybrid. 2- Fodder maize. J. Agric. Sci. Mansoura Univ., 22 (12): 41154123.

Sanderson, M.A., R.M. Jones, J.C. Read and H. Lippke (1995). Digestibility and lignocellulose composition of forage corn morphological components. J. Prod. Agric. USA, 8: 169-174.

Soleymani, A. and M. H. Shahrajabian (2013). The effects of plant densities on LAI and dry matter of different parts of sweet sorghum cultivars. Int. J. Agron. and Plant Prod., 2 (2): 84-88.

Steel, R.G.D., J.H. Torrie and D.A. Dickey (1997). Principles and Procedures of Statistics. A Biometrical Approach, $3^{\text {rd }}$ Ed. McGraw Hill.

Turgut, I., U. Bilgili, A. Duman and E. Acikgoz (2005). Production of sweet sorghum (Sorghum bicolor L. Moench) increases with increased plant densities and nitrogen fertilizer levels. Acta Agriculturae Scandinavica, Section BSoil and Plant Sci., 35 (3): 236 - 240.

Yousef, M.S.H.A. (2002). Effect of nitrogen fertilization and plant spacing on forage sorghum under new reclaimed land conditions. Agric., Res. J. Suez Canal, Univ., $1: 15-22$. 
تأثير كثافة الزراعة ومستويات السماد النيتروجينى على كمية محصول العلف الأخضر وصفات الجودة

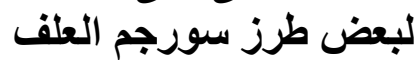

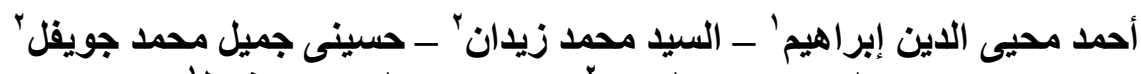

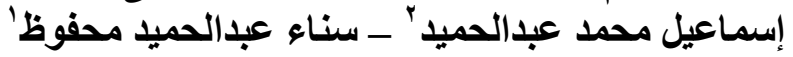

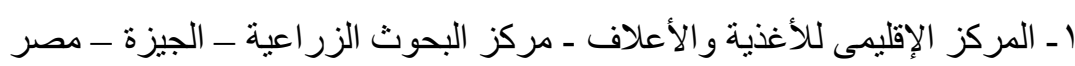

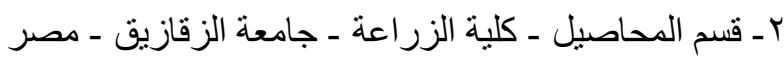

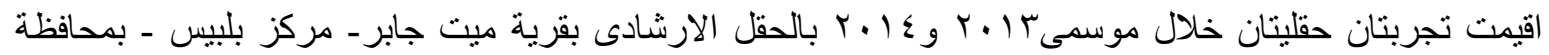

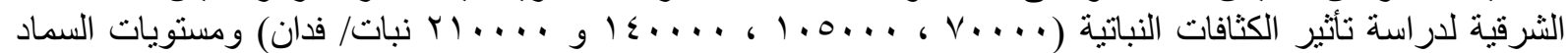

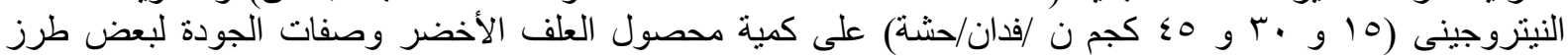

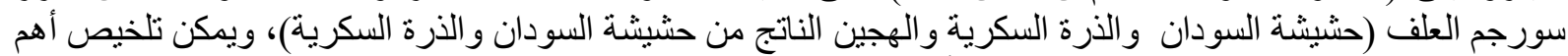

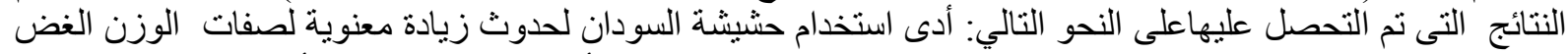

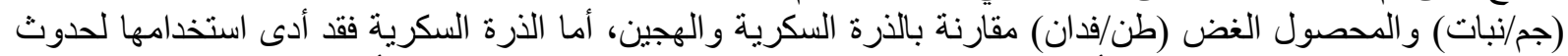

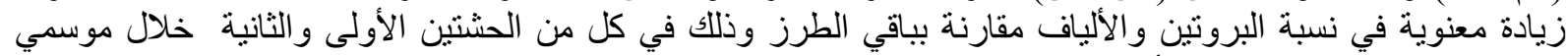

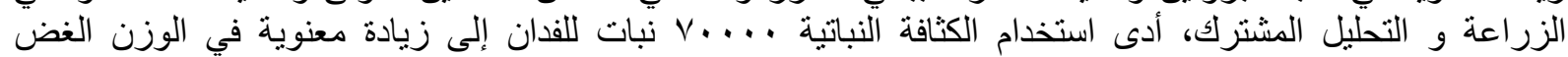

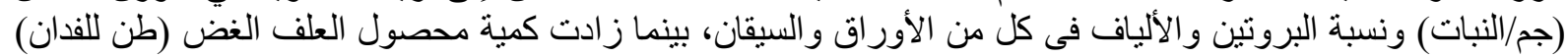

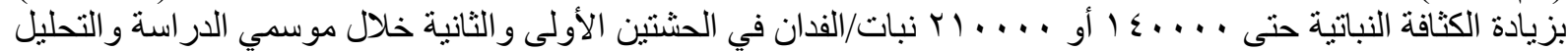

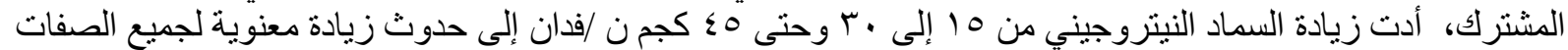

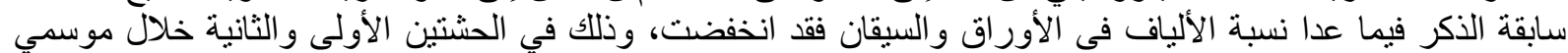

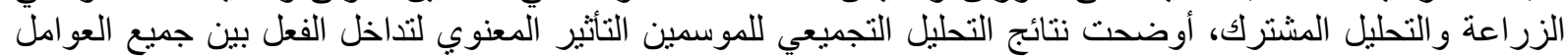

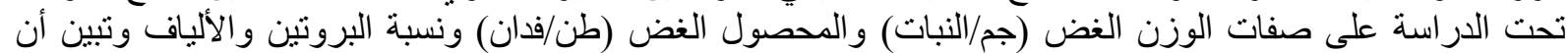

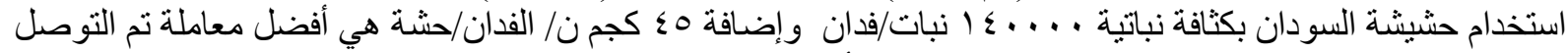

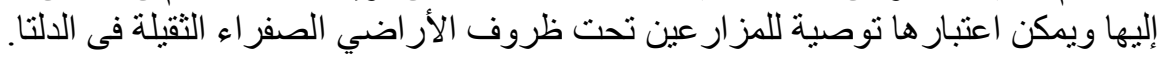

